\title{
Moving forward during major goal blockage: situational goal adjustment in women facing infertility
}

\author{
Elizabeth H. Thompson • Julia T. Woodward • \\ Annette L. Stanton
}

Received: June 2, 2010/Accepted: December 9, 2010/Published online: January 8, 2011

(c) The Author(s) 2010. This article is published with open access at Springerlink.com

\begin{abstract}
Individuals confronting chronic medical conditions often face profound challenges to cherished life goals. The primary aim of this study was to examine the associations of goal adjustment with psychological adjustment in the context of infertility. At study entry (T1; $\mathrm{n}=97)$ and 6 months later $(T 2 ; n=47)$, women in fertility treatment completed measures of goal blockage, goal adjustment ability, and psychological adjustment. At T1, greater perceived and actual goal blockage were related to negative psychological adjustment. Ability to disengage from the goal of biological parenthood was associated with less infertility-specific thought intrusion, whereas engagement with other goals was related to fewer depressive symptoms and greater positive states of mind. Greater general goal engagement was protective against the negative relationships between low goal disengagement and the dependent variables. Promoting letting go of the unattainable and investing in the possible may be a useful intervention to foster well-being among individuals experiencing profound goal blockage.
\end{abstract}

Keywords Goal blockage - Goal adjustment . Chronic disease $\cdot$ Infertility $\cdot$ Self-regulation

E. H. Thompson · A. L. Stanton ( $\square)$

Department of Psychology, University of California, Los Angeles, 1285 Franz Hall, Box 951563, Los Angeles, CA 90095-1563, USA

e-mail: astanton@ucla.edu

J. T. Woodward

Department of Psychiatry and Behavioral Sciences, Duke U.

Medical Center, Duke Fertility Center, Durham, NC, USA

\section{Introduction}

Western society frowns upon "quitters," celebrating instead individuals who demonstrate persistence and determination (Dougher 1994). But what happens when an individual confronts a goal that might be permanently blocked? Chronic medical conditions can impede numerous goals, necessitating marked alteration of one's plans for the future. Researchers (e.g., Carver and Scheier 2000; Heckhausen et al. 2010; Wrosch 2011) assert that disengagement from such blocked goals is adaptive, as it releases the individual from a futile effort and allows engagement in new or formerly set-aside goals. Research has supported the hypothesis that goal disengagement ability, as assessed as a dispositional tendency, is psychologically and physiologically adaptive (Miller and Wrosch 2007; Wrosch et al. 2007). Reengagement in alternative goals is related to positive psychological adjustment to goal blockage, and goal reengagement can buffer against low goal disengagement ability (Heckhausen et al. 2010; Wrosch et al. 2003b, 2008).

Although the benefits of goal disengagement and reengagement have been demonstrated, this emerging body of research is limited in several ways. First, studies have often employed a dispositional measure of goal adjustment ability rather than a situational measure assessing response to a current goal blockage. Such an approach does not allow examination of the extent of objective and subjective goal blockage or of the influence of engagement in extant valued goals and the generation of new goals on adjustment. Second, many studies have been conducted with healthy, young participants, who may never have experienced profound threat to a central life goal (Wrosch et al. 2003b). Third, little research has addressed psychological factors that contribute to these self-regulation strategies. 
The primary goals of this study are to examine the relationships among situational goal adjustment processes, perceived and actual goal blockage, and psychological adjustment in the context of infertility and to specify factors that promote or inhibit goal adjustment. In addition to carrying theoretical implications, understanding the relationships between goal perceptions and goal adjustment in the context of a chronic medical stressor can aid clinicians in facilitating adaptive goal adjustment and promoting the pursuit of life goals to buffer the negative psychological effects associated with goal blockage. Such research can enable identification of risk factors for poor adjustment as a result of inflexible approaches to goal pursuit.

Wrosch et al. (2007) proposed a theory of adaptive goal adjustment based on two self-regulation strategies: goal disengagement, which reduces distress and avails psychological resources, and goal reengagement, which increases well-being. Likewise, Heckhausen et al.'s (2010) motivational theory of life-span development employs a developmental framework to examine goal adjustment constructs that overlap with Wrosch's model. Goal disengagement is characterized by reducing effort toward goal completion and commitment to the goal, whereas goal reengagement consists of identifying an alternative goal, committing to the goal, and commencing activities towards achieving it (Wrosch and Scheier 2003; Wrosch et al. 2003a). Dispositional goal disengagement ability is associated with high self-mastery, low perceived stress, low intrusive thoughts, fewer physical health problems (Wrosch et al. 2003b), more normative (steeper) diurnal cortisol rhythms, better sleep efficiency (Wrosch et al. 2007), and lower levels of the inflammatory marker C-reactive protein (Miller and Wrosch 2007). Disengagement and reengagement processes can interact, such that reengagement buffers the negative effects of low disengagement on adjustment (Wrosch et al. 2003b). Theories proposed by Wrosch et al. (2007) and Heckhausen et al. (2010) both emphasize the adaptive nature of altering personal goals when situational factors and biological constraints make goal achievement unlikely.

Research demonstrates that dispositional disengagement and reengagement abilities are independent processes, and these processes may have unique relationships with positive and negative measures of adjustment (Thompson and Stanton 2009; Wrosch 2011). Goal disengagement may be associated with a reduction in negative affect, rumination, intrusive thoughts and depressive symptoms, whereas goal reengagement may be associated with an increase in positive affect, sense of purpose, and well-being, as individuals experience progress towards an attainable goal. The majority of research on goal adjustment has focused on negative outcomes (e.g., depressive symptoms, intrusive thoughts), but more recent research, including the current study, incorporates measures of positive adjustment.

Infertility is a context well suited to understanding processes of goal adjustment, in that it constitutes a potentially profound blockage of a central life goal, and women receiving treatment frequently receive feedback on their goal progress. Although having a biological child is a major life goal for many adults, becoming pregnant can be difficult or impossible. Affecting more than $10 \%$ of married couples (Abma et al. 1997), infertility is defined as the inability to conceive a child after 1 year of engaging regularly in unprotected sexual intercourse (Mosher and Pratt 1982). In addition, $15 \%$ of pregnancies end in miscarriage (Hill 1995). When the goal of biological parenthood is blocked, often after years of trying to prevent conception, couples can experience depression and frustration (Clark et al. 1991; Greil 1997). Clark et al. (1991) noted that "the dilemma of being caught between goal-fulfillment strivings and acceptance of one's goal blockage or permanent infertility may be the most stressful aspect of coping with infertility" (p. 166). In line with theories of goal adjustment as an adaptive self-regulation strategy (Heckhausen et al. 2010; Wrosch et al. 2007; see Woodward 2004, for a situational theory), our primary hypotheses were that greater disengagement from the goal of biological parenthood would be associated with fewer depressive symptoms and less infertility-related thought intrusion, and that goal engagement in direct response to infertility and general goal engagement both would be associated with more positive states of mind.

Examining goal-related processes in a situational context allows consideration of the extent of goal blockage on adaptive outcomes. During infertility treatment, women often receive monthly objective feedback on goal progress in the form of a positive or negative pregnancy test. Because high perceived goal blockage in conjunction with continued infertility treatment represents goal striving without goal attainment, we hypothesized it to be related to negative adjustment. While this hypothesis has been supported by previous data, objective measures of goal blockage have not been studied. Actual goal blockage in infertility can be operationalized as number of discrete unsuccessful treatment attempts. In that high actual goal blockage represents emotional, behavioral, and financial commitment to parenthood without goal progress, we hypothesized that it would also be associated with negative adjustment.

This study expands upon two previous studies that have examined situational goal processes in the context of infertility. Salmela-Aro and Suikkari (2008) examined ratings of goal importance and goal attainability in a sample of women and men receiving infertility treatment. Six months after treatment failure, high ratings of goal 
importance (assessed by a single item) and low ratings of goal attainment were significantly associated with depressive symptoms. The authors interpreted findings as indicating that goal disengagement is an adaptive response to prolonged infertility. The current study builds on these findings by including reports of cognitive and behavioral aspects of goal disengagement (rather than using goal importance as a proxy for goal disengagement), as well as assessing general goal engagement and goal engagement in direct response to infertility, which are unique aspects of goal adjustment that may relate to psychological adjustment in distinctive ways.

In cross-sectional analyses of 83 men and women who had been aware of definitive infertility problems for an average of 12 years, Kraaij et al. (2009) found that disengagement from the goal of biological parenthood was related to low negative affect, whereas goal reengagement was related to positive affect. The interaction between goal disengagement and goal reengagement was not reported, and the generalizability of these findings is limited due to the unique sample studied. The current study allows for comparison between alternate goal engagement independent of the goal blockage and engagement that occurs in direct response to goal blockage. Motivation for pursuing an alternate goal may be pre-existing and unprompted, or it may be incited by experiencing goal blockage. For example, one infertile woman may be focused on an already satisfying career (alternate goal engagement), whereas another woman might have assumed that motherhood would constitute her career and seek other options only after experiencing infertility (goal engagement in direct response to infertility). Role accumulation theory (Sieber 1974) asserts that having multiple valued roles is protective for women's mental and physical health, and empirical data support this theory (Barnett 2004; Ruderman et al. 2002). Heckhausen et al. (2010) highlight the protective value of goal diversity and posit that maintaining varied goals is an essential component of adaptive goal pursuit. Linville also posits that greater selfcomplexity buffers against depressive and physical symptoms in response to stress, and evidence supports this theory specifically in the context of failed goals (Linville 1987; Niedenthal et al. 1992).

Relative to women who lack other meaningful life pursuits, women who endorse high engagement with preexisting goals in areas such as careers or relationships may be at lower risk for depressive symptoms or thought intrusion and may be more likely to experience positive affect as they make progress toward these alternative goals. The current study employs a situational measure that asks participants to evaluate their goal adjustment in direct response to their infertility, as well as their level of engagement with pre-existing goals, in order to test whether they evidence similar relations with adjustment.
Which psychological factors promote or impede an individual's ability to accept immutable goal blockage and pursue new goals? In a sample of 97 adolescent girls, high initial depressive symptoms predict an increase in dispositional goal disengagement ability and no significant change in reengagement ability over 13 months (Wrosch and Miller 2009). The authors noted that the generalizability of this finding may be limited, as the sample was young, female, and identified as being at high risk for developing depressive episodes. Heckhausen et al. (2010) point to empirical evidence indicating that goal appraisal and goal adjustment strategies change over the lifespan. During adolescence, depressive symptoms may serve the adaptive function of promoting the improvement of goal disengagement ability, but when experienced in adulthood, the associated cognitive rigidity and apathy may hinder individuals' abilities to let go of cherished goals and reinvest in new goals (Wrosch and Miller 2009). In the present sample, depression might hinder women's abilities to disengage and enthusiastically reengage in alternate goals. We examined this question as well as additional potential contributors to situational goal adjustment ability. We postulated that greater infertility-specific intrusive thoughts would predict a decrease in goal disengagement and goal engagement, as women experiencing intrusive thoughts would be likely to remain fixated on the blocked goal. We also expected that greater positive states of mind would predict an increase in goal engagement, as experiencing positive states may promote flexibility and motivate women to pursue new endeavors (Frederickson 2001). We hypothesized that both high actual and perceived goal blockage would predict an increase in goal disengagement, as individuals appraise the attainment of biological parenthood as increasingly unlikely.

\section{Methods}

Recruitment was conducted in a fertility clinic at an academic medical center in the Midwest. Women were eligible if they were seeking fertility treatment and able to read and write in English. Women who were visiting the clinic for the first time or who were seeking artificial insemination by a sperm donor and did not have an infertility diagnosis were not recruited. Of 112 women introduced to the study, 97 elected to participate, 10 declined, and five consented but did not complete the initial questionnaire (T1), representing $87 \%$ participation. An independent samples $t$-test revealed no significant difference in the age of participants $(M=$ 33.13 years, $\mathrm{SD}=5.57)$ and decliners $(M=29.80$ years, $\mathrm{SD}=5.90 ; t[103]=1.79, P>.05)$. Fifty-eight women completed an additional assessment 6 months later (T2). Four participants were lost to follow up, and the remaining 
participants did not return the assessment materials. Of the women who completed T2, 11 had become pregnant since T1. The 47 who were not pregnant were retained in analyses. No significant differences emerged on demographic or fertility-related variables between completers and noncompleters.

The 97 participants had a mean educational level of 15.15 years $(\mathrm{SD}=2.4) ; 70 \%$ were Caucasian, $19 \%$ African American, 4\% Latina, 3\% Asian American, 1\% Native American, and $3 \%$ were of other ethnic backgrounds. Most women (90\%) were married, for an average of 6.50 years $(\mathrm{SD}=4.28)$, and $64 \%$ had no children. Most women $(71 \%)$ reported that they had been diagnosed with a fertility problem, $8 \%$ reported that their partner had been diagnosed, $7 \%$ reported that both they and their partner had been diagnosed, and 14\% reported infertility of unknown cause. On average, participants had been trying to get pregnant for more than 2 years $(M=26.72$ months; $\mathrm{SD}=33.90 ;$ range $=0-240)$ prior to seeking fertility treatment. The average duration of fertility treatment was 18.06 months $\quad(\mathrm{SD}=21.36$; range $=0-132$ ). Fifty-one percent of the women had taken Clomid for at least one cycle, $12 \%$ had intrauterine insemination (IUI), $27 \%$ had IUI with Clomid, $21 \%$ had IUI with an injectable fertility medication, $16 \%$ had in vitro fertilization (IVF), and $10 \%$ had other infertility treatment. Thirty-four percent were in the diagnostic phase and had not yet undergone treatment procedures. Sixteen percent reported having at least one miscarriage before receiving fertility treatment; $15 \%$ reported miscarrying at least once during treatment.

\section{Procedure}

Study procedures were approved by the Institutional Review Board of the University of Kansas Medical Center. Patients meeting eligibility criteria were approached in the fertility clinic waiting room by the second author (JTW) and invited to participate in a study examining "how patients who have been diagnosed with fertility problems learn to deal with that experience." The two clinic physicians provided prior approval for their patients to be approached, and they were unaware of whether patients agreed to participate. After providing written informed consent, participants completed questionnaires individually in a quiet room in the clinic. Six months later, follow-up questionnaires were completed by mail.

\section{Measures}

Goal adjustment in the context of infertility was assessed with an author-constructed self-report measure. Nineteen items were generated by the second author to reflect goal disengagement or engagement abilities (Table 1). Participants were instructed, "Please turn your attention to your goal of becoming a parent. Answer the following questions with that goal in mind. If you already have children, think about your current goal of having a child." Items were answered on a 1-7 scale from completely disagree to completely agree. Although items are specific to infertility, the scale was designed so that it can be adapted to other populations. The 19 items administered at study entry were submitted to a principal axis factor analysis with Promax rotation to allow the factors to be correlated. A three-factor solution best characterized the data, accounting for $56 \%$ of the variance, as indicated by three criteria: (1) eigenvalues greater than 1.0, (2) relatively high factor loadings of items on their respective factors $(>.50)$, and (3) relatively low loadings on the other factors $(<.30)$. Accounting for $36 \%$ of the variance, the first factor contained nine items that reflect goal disengagement ability. Comprised of three items accounting for $13 \%$ of the variance, the second factor reflected new goal pursuit in direct response to infertility. The third factor contained three items reflecting alternative goal pursuit unprompted by infertility and accounted for $7 \%$ of the variance. Four items did not load on any single factor. Items were averaged to construct the resulting three scales, with internal consistency estimates for the three scales at $\alpha=.92$ for goal disengagement, .88 for goal engagement in direct response to infertility, and .89 for general goal engagement. The correlation of the two goal engagement scales was $r=.35(P<.0005)$, and goal disengagement ability correlated with engagement in direct response to infertility at $r=.41, P<.0001$ and with general goal engagement at $r=.15, P>.10$.

Goal blockage was assessed by a self-report item ("How blocked do you feel in your goal of becoming a parent?" answered on a 1-7 scale from not blocked to completely blocked) termed Perceived Goal Blockage, and by the total number of unsuccessful treatment cycles the participant had undergone, termed Actual Goal Blockage.

Depressive symptoms were assessed with the 20-item Center for Epidemiologic Studies Depression Scale (CES-D; Radloff 1977, 1991). This scale has good internal consistency with both clinical and general adult populations ( $\alpha$ of .90 and .85 , respectively; Radloff 1991).

Infertility-specific thought intrusion was assessed using the 7-item Intrusion subscale of the Impact of Event Scale (Horowitz et al. 1979). Participants were asked how frequently each item was true for them with regard to their experience of fertility problems during the past week $(0=$ Not at all; $5=$ Often $)$. A sample item is, "Other things kept making me think about it." This scale has adequate internal consistency $(\alpha=.78$ for the Intrusion 
Table 1 Promax rotated factor loadings from the principal axis factor analysis

\begin{tabular}{|c|c|c|c|}
\hline Item & Factor 1 & Factor 2 & Factor 3 \\
\hline It would be difficult to let go of my dream of becoming a parent ${ }^{\mathrm{a}}$ & 0.74 & -0.13 & 0.16 \\
\hline I long for my own baby ${ }^{a}$ & 0.73 & -0.08 & 0.09 \\
\hline When I see others with their children, it makes me want to become a parent ${ }^{\mathrm{a}}$ & 0.68 & -0.23 & 0.15 \\
\hline It would be difficult for me to give up my goal of becoming a parent ${ }^{\mathrm{a}}$ & 0.77 & -0.05 & 0.01 \\
\hline I am focused on having a child ${ }^{\mathrm{a}}$ & 0.84 & 0.13 & -0.10 \\
\hline I will try for as long as it takes to become a parent ${ }^{\mathrm{a}}$ & 0.73 & 0.10 & -0.01 \\
\hline I spend much of my energy trying to have a child ${ }^{\mathrm{a}}$ & 0.75 & 0.02 & 0.10 \\
\hline Nothing will stop me from to the goal of having a child ${ }^{\mathrm{a}}$ & 0.70 & 0.05 & 0.05 \\
\hline Eventually I could make peace with not becoming a parent. & 0.51 & 0.18 & 0.24 \\
\hline I have other meaningful goals besides becoming a parent. & 0.04 & 0.11 & 0.82 \\
\hline I am pursuing other meaningful goals besides becoming a parent. & 0.07 & 0.08 & 0.81 \\
\hline There are other goals in my life that are just as important as parenthood. & 0.18 & 0.07 & 0.65 \\
\hline Because of our fertility problems, I have developed new goals. & -0.14 & 0.70 & 0.15 \\
\hline Our fertility problems have led me to focus again on former interests. & -0.17 & 0.81 & 0.11 \\
\hline Because of our fertility problems, I am investing more time in other goals. & -0.02 & 0.76 & 0.21 \\
\hline
\end{tabular}

Standardized regression coefficients are reported. Four items with low factor loadings are not included

${ }^{\text {a }}$ Reverse-scored

subscale) and high test-retest reliability ( $r=.87$; Horowitz et al. 1979).

Positive states of mind were assessed using the six-item Positive States of Mind Scale (PSOM; Horowitz et al. 1988), which taps current life satisfaction and ability to experience positive states such as productivity, pleasure and connection to others. The scale has a Cronbach $\alpha$ of .77 , is positively correlated with the Vigor subscale of the Profile of Mood States and is negatively correlated with negative mood (Horowitz et al. 1988).

\section{Statistical analyses}

Bivariate correlations between goal adjustment and psychological adjustment variables were calculated. Dependent $t$-tests were used to assess for change in goal adjustment and psychological adjustment measures. $T$-tests, $\chi^{2}$ (for categorical variables) and correlations (for continuous variables) with the psychological adjustment variables were used to select demographic (i.e. age, years of education, ethnicity coded as Caucasian [0] and minority [1], marital status coded as married [0] and single, divorced, or widowed [1], number of years married, and whether women had any children) and infertility-related covariates (i.e. number of unsuccessful treatment attempts, number of months trying to conceive before receiving treatment, number of months in treatment, and whether women had experienced a miscarriage) for use in primary regression analyses. Measures of psychological adjustment were regressed on goal blockage and adjustment measures, and the interactions between goal disengagement and both measures of goal engagement at T1 and at T2 (controlling for $\mathrm{T} 1$ psychological adjustment). To examine hypotheses regarding psychological factors that may hinder or aid goal adjustment, T2 goal adjustment measures were regressed on T1 psychological adjustment measures controlling for T1 goal adjustment.

\section{Results}

Descriptive statistics

Table 2 displays descriptive data and internal reliability estimates for the measures. The CES-D mean at T1 equaled the cutoff score of 16 suggestive of clinical depression (Radloff 1977). On average, women reported that they "sometimes" experienced intrusive thoughts related to infertility. The intrusion means were similar to those Cordova et al. (2001) reported for women with Stages 0 -IIIB breast cancer who were on average 2 years after treatment completion $(M=11.1, \mathrm{SD}=9.0)$. The PSOM mean was higher than that reported by Horowitz et al. (1988) for undergraduate women $(M=12.2, \mathrm{SD}=3.5)$. On average, women reported minor limitations in doing things that led to positive states of mind. On average, participants had experienced 4.15 unsuccessful fertility treatments and felt moderately blocked in their goal of becoming a parent at T1. Dependent t-tests revealed no significant change from $\mathrm{T} 1$ to $\mathrm{T} 2$ for goal adjustment and psychological adjustment measures, although substantial individual variability in scores was evident. Correlations 
Table 2 Descriptive statistics for goal measures and psychological adjustment variables

\begin{tabular}{|c|c|c|c|c|c|}
\hline & $\mathrm{N}$ & Mean & SD & Range & Alpha \\
\hline Time 1 Disengagement Ability & 95 & 2.79 & 1.35 & $1.00-6.56$ & .92 \\
\hline Time 1 Engagement in Response to Infertility & 97 & 3.40 & 1.60 & $1.00-7.00$ & .88 \\
\hline Time 1 General Goal Engagement & 97 & 4.57 & 1.70 & $1.00-7.00$ & .89 \\
\hline Time 2 Disengagement Ability & 46 & 2.90 & 1.12 & $1.00-5.89$ & .89 \\
\hline Time 2 Engagement in Response to Infertility & 46 & 3.42 & 1.50 & $1.00-6.00$ & .90 \\
\hline Time 2 General Goal Engagement & 46 & 4.45 & 1.59 & $1.00-7.00$ & .87 \\
\hline Time 1 Actual Goal Blockage & 94 & 4.15 & 5.99 & $0.00-44.00$ & - \\
\hline Time 1 Perceived Goal Blockage & 96 & 3.95 & 1.91 & $1.00-7.00$ & - \\
\hline Time 1 CES-D & 96 & 16.07 & 12.18 & $0.00-52.00$ & .93 \\
\hline Time 1 INT & 96 & 11.92 & 8.46 & $0.00-35.00$ & .82 \\
\hline Time 1 PSOM & 97 & 14.58 & 3.29 & $6.00-18.00$ & .84 \\
\hline Time 2 CES-D & 46 & 17.67 & 13.65 & $0.00-44.00$ & .94 \\
\hline Time 2 INT & 46 & 11.54 & 8.77 & $0.00-31.00$ & .83 \\
\hline Time 2 PSOM & 47 & 14.02 & 4.11 & $2.00-18.00$ & .92 \\
\hline
\end{tabular}

$C E S-D$ Center for Epidemiologic Studies-Depression Scale, INT Infertility-specific thought intrusion as measured by IES (Intrusion subscale), PSOM Positive States of Mind scale

among the goal adjustment subscales and psychological adjustment variables at $\mathrm{T} 1$ and $\mathrm{T} 2$ are shown in Table 3. Actual goal blockage (number of treatment attempts) and perceived goal blockage were uncorrelated both when all participants were included $(r=-.09)$, and when women who reported no treatment attempts were excluded $(r=$ $-.17)$.

Psychological adjustment regressed on goal adjustment abilities

As the only demographic or infertility-related variable significantly related to any $\mathrm{T} 1$ dependent variable, treatment duration was entered as a covariate for the CES-D $(r=.21, P<.05)$. As displayed in Table 4 , the overall model testing goal blockage, goal adjustment, and the interactions between goal adjustment measures as predictors of depressive symptoms accounted for $18 \%$ of its variance, $F(8,81)=3.36, P<.05$. Greater perceived goal blockage was associated with more depressive symptoms. The significant association between greater general goal engagement and lower depressive symptoms was qualified by a significant interaction between goal disengagement and general goal engagement. The relationships between general goal engagement and depressive symptoms at the mean and one standard deviation above and below the mean of goal disengagement are illustrated in Fig. 1. An analysis of simple slopes indicated that for women reporting average or low levels of disengagement, general goal engagement was associated with lower depressive symptoms (Mean disengagement: $\beta=-.24, t=-2.03$,
$P<.05 ;-1$ SD disengagement $\beta=-.46, t=-2.87$, $P<.01$ ), but for women reporting high levels of disengagement, general goal engagement was not significantly associated with depressive symptoms $(\beta=-.04, t=-.29$, $P=.78)$. Predicted CES-D scores for women with average or low disengagement ability coupled with low general goal engagement exceed 20.

The overall model for intrusive thoughts explained $22 \%$ of its variance $F(7,83)=4.55, P<.001$ (Table 4). Higher perceived and actual goal blockage were associated significantly with having more intrusive thoughts. The relation of greater goal disengagement ability with fewer intrusive thoughts was qualified by a significant interaction between goal disengagement and general goal engagement, illustrated in Fig. 2. Simple slopes analyses were not significant at the mean of goal disengagement $(\beta=-.08, t=-.72$, $P=.47)$ or at one SD above $(\beta=.11, t=.73, P=.47)$ the mean, but approached significance at one SD below the mean $(\beta=-.28, t=-1.84, P=.07)$. For women who reported low disengagement, the relationship between general goal engagement and intrusive thoughts was negative, whereas for women who reported high disengagement, the relationship between general goal engagement and intrusive thoughts was positive. These relationships were significantly different from each other, but neither slope was significantly different from zero. Low disengagement ability in combination with low general goal engagement was associated with the highest infertilityspecific intrusive thoughts.

The overall model for positive states of mind (Table 4) accounted for $22 \%$ of its variance $F(7,84)=4.71$, 
Table 3 Correlations of time $1(n=94)$ and time $2(n=47)$ variables

\begin{tabular}{|c|c|c|c|c|c|c|c|c|c|c|c|c|c|}
\hline & 1 & 2 & 3 & 4 & 5 & 6 & 7 & 8 & 9 & 10 & 11 & 12 & 13 \\
\hline \multicolumn{14}{|l|}{$\begin{array}{l}\text { 1. T1 Disengagement } \\
\text { Ability }\end{array}$} \\
\hline $\begin{array}{l}\text { 2. T1 Engagement in } \\
\text { Response to } \\
\text { Infertility }\end{array}$ & .14 & & & & & & & & & & & & \\
\hline $\begin{array}{l}\text { 3. T1 General Goal } \\
\text { Engagement }\end{array}$ & $.47 * *$ & $.35^{*}$ & & & & & & & & & & & \\
\hline $\begin{array}{l}\text { 4. T2 Disengagement } \\
\text { Ability }\end{array}$ & $.69 * *$ & $.37 *$ & $.35^{*}$ & & & & & & & & & & \\
\hline $\begin{array}{l}\text { 5. T2 Engagement in } \\
\text { Response to } \\
\text { Infertility }\end{array}$ & .04 & $.46 * *$ & $.49 * *$ & .20 & & & & & & & & & \\
\hline $\begin{array}{l}\text { 6. T2 General Goal } \\
\text { Engagement }\end{array}$ & $.35^{*}$ & .26 & $.70 * *$ & $.39 * *$ & $.52 * *$ & & & & & & & & \\
\hline $\begin{array}{l}\text { 7. T1 Actual Goal } \\
\text { Blockage }\end{array}$ & -.06 & .04 & -.10 & -.24 & .08 & -.04 & & & & & & & \\
\hline $\begin{array}{l}\text { 8. T1 Perceived Goal } \\
\text { Blockage }\end{array}$ & $.37 * *$ & .10 & -.18 & .05 & -.13 & $-.29 *$ & -.09 & & & & & & \\
\hline 9. T1 CES-D & $-.23 *$ & -.12 & $-.26^{*}$ & $-.43 * *$ & -.05 & $-.40 * *$ & .15 & $.25^{*}$ & & & & & \\
\hline 10. T1 INT & $-.39 * * *$ & -.10 & $-.22 *$ & $-.45 * *$ & .07 & $-.44 * *$ & .17 & $.29 * *$ & $.70 * * *$ & & & & \\
\hline 11. T1 PSOM & .20 & $.22 *$ & $.30 * *$ & $.41 * *$ & .02 & $.36^{*}$ & -.18 & $-.26 *$ & $-.80 * *$ & $-.60 * *$ & & & \\
\hline 12. T2 CES-D & -.21 & -.26 & $-.44 * *$ & $-.35^{*}$ & -.19 & $-.63 * *$ & .11 & $.29 *$ & $.69 * *$ & $.69 * *$ & $-.71 * *$ & & \\
\hline 13. T2 INT & $-.37 *$ & -.21 & $-.37 *$ & $-.48 * *$ & -.08 & $-.66^{* *}$ & .11 & .23 & $.60 * *$ & $.74 * *$ & $-.54 * *$ & $.84 * * *$ & \\
\hline 14. T2 PSOM & -.05 & .18 & .29 & $.33 *$ & .12 & $.57 * *$ & -.18 & -.10 & $-.69 * *$ & $-.68 * *$ & $.75 * *$ & $-.83 * *$ & $-.77 * *$ \\
\hline
\end{tabular}

$C E S-D$ Center for Epidemiologic Studies-Depression Scale, INT Infertility-specific thought intrusion as measured by IES (Intrusion subscale), PSOM Positive States of Mind scale

$* P<.05 ; * * P<.01 ; * * * P<.001$

$P<.001$. Higher perceived actual and goal blockage were associated significantly with lower positive states of mind. The significant relation of greater general goal engagement with more positive states of mind was qualified by a significant interaction between goal disengagement and general goal engagement, illustrated in Fig. 3. Simple slopes analyses revealed that for women who reported mean levels or lows levels of disengagement ability, general goal engagement was associated with greater positive states of mind (Mean disengagement: $\beta=.24, t=2.09, P<.05$; -1 SD disengagement $\beta=.47, t=3.25, P<.01$ ), but for women who reported high levels of disengagement, general goal engagement was not significantly associated with positive states of mind ( $+1 \mathrm{SD}: \beta=.01, t=.06, P=.95$ ). The combination of low disengagement ability and low general goal engagement was associated with particularly low positive states of mind.

Regression analyses were also conducted to examine whether goal adjustment measures at $\mathrm{T} 1$ were associated with change in psychological adjustment across time in the smaller subsample. In Step 1, T1 psychological adjustment measures and covariates (i.e. treatment duration for T2 depressive symptoms $[r=.33, P<.05]$ and greater education for T2 intrusive thoughts $[r=.30, P<.05])$ were controlled and in following steps, goal-related predictor variables and interactions were entered to predict T2 psychological adjustment. Study entry levels of psychological adjustment variables and covariates accounted for $45-57 \%$ of the variation in T2 psychological adjustment (all $P<.001$ ), and none of the goal adjustment measures emerged as significant predictors of change in psychological adjustment over and above T1 levels and covariates. ${ }^{1}$

\footnotetext{
${ }^{1}$ In response to reviewers' recommendations, additional analyses were conducted to assess for group difference between women with children $(\mathrm{N}=35)$ and women without children $(\mathrm{N}=62)$. On average, women with children had been married for a longer time than women without children $(t[80]=-2.88, P<.01)$, but no other differences on demographics or infertility-specific variables emerged. Controlling for differences in number of years married, ANCOVAs indicated no significant group differences on psychological adjustment indices, goal blockage variables, or goal engagement in direct response to infertility at $\mathrm{T} 1$. Women with children reported significantly higher goal disengagement (T1, T2) and general goal engagement (T1) than women without children. Findings from analyses examining the hypothesized association between psychological adjustment variables and interactions between goal disengagement and general goal engagement were not affected significantly by whether women did or did not have children (controlling for years
} 
Table 4 Goal adjustment as related to psychological adjustment at study entry

\begin{tabular}{|c|c|c|c|c|c|c|}
\hline $\mathrm{DV}$ & Step & IV & $R^{2} \Delta$ & $B$ & $\mathrm{SE}(B)$ & $\beta$ (final) \\
\hline \multirow[t]{8}{*}{ CES-D T1 ${ }^{\mathrm{a}}$} & 1 & Treatment length & $.05^{*}$ & 0.14 & 0.06 & $.24 *$ \\
\hline & \multirow[t]{2}{*}{2} & Actual goal blockage & \multirow[t]{2}{*}{$.07 *$} & 0.19 & 0.24 & .10 \\
\hline & & Perceived goal blockage & & 1.62 & 0.64 & $.26^{*}$ \\
\hline & \multirow[t]{3}{*}{3} & GD & \multirow[t]{3}{*}{.07} & -0.03 & 0.12 & -.03 \\
\hline & & GEINF & & -0.01 & 0.28 & -.00 \\
\hline & & GRGEN & & -0.65 & 0.29 & $-.27 *$ \\
\hline & \multirow[t]{2}{*}{4} & GD x GEINF & \multirow[t]{2}{*}{.05} & 0.01 & 0.24 & .05 \\
\hline & & GD x GEGEN & & 0.04 & 0.02 & $.22 *$ \\
\hline \multirow[t]{7}{*}{ INT $\mathrm{T}^{\mathrm{b}}$} & \multirow[t]{2}{*}{1} & Actual goal blockage & \multirow[t]{2}{*}{$.11^{* *}$} & 0.30 & 0.14 & $.22 *$ \\
\hline & & Perceived goal blockage & & 1.29 & 0.43 & $.30 * * *$ \\
\hline & \multirow[t]{3}{*}{2} & GD & \multirow[t]{3}{*}{$.10^{*}$} & -0.18 & 0.08 & $-.27 *$ \\
\hline & & GEINF & & 0.01 & 0.19 & .01 \\
\hline & & GEGEN & & -0.20 & 0.20 & -.12 \\
\hline & \multirow[t]{2}{*}{3} & GD x GEINF & \multirow[t]{2}{*}{$.06^{*}$} & 0.01 & 0.02 & .09 \\
\hline & & GD x GEGEN & & 0.03 & 0.01 & $.21^{*}$ \\
\hline \multirow[t]{7}{*}{ PSOM T1 $1^{\mathrm{c}}$} & \multirow[t]{2}{*}{1} & Actual goal blockage & \multirow[t]{2}{*}{$.09 * *$} & -0.12 & 0.06 & $-.22 *$ \\
\hline & & Perceived goal blockage & & -0.45 & 0.17 & $-.26^{*}$ \\
\hline & \multirow[t]{3}{*}{2} & GD & \multirow[t]{3}{*}{$.10^{*}$} & -0.01 & 0.03 & -.03 \\
\hline & & GEINF & & 0.09 & 0.07 & .13 \\
\hline & & GEGEN & & 0.18 & 0.08 & $.27 *$ \\
\hline & \multirow[t]{2}{*}{3} & GD x GEINF & \multirow[t]{2}{*}{$.07 *$} & -0.00 & 0.01 & -.06 \\
\hline & & GD x GEGEN & & -0.01 & 0.00 & $-.26^{*}$ \\
\hline
\end{tabular}

CESD Center for Epidemiologic Studies-Depression Scale, INT Infertility-specific thought intrusion as measured by IES (Intrusion subscale), PSOM Positive States of Mind scale, GD Goal disengagement, GEINF Goal engagement in response to infertility, GEGEN Goal engagement without reference to infertility. $R$ reported is adjusted $R^{2}$

$* P<.05 ; * * P<.01 ; * * * P<.001$

${ }^{\mathrm{a}} n=90$

b $n=91$

c $n=92$

Footnote 1 continued

married). Analyses examining the hypothesized relationship between goal appraisals and psychological adjustment revealed that number of treatment attempts (actual goal blockage) was associated with poorer psychological adjustment for women with children but was not significantly associated with psychological adjustment for women without children. This finding may be explained by the greater variation in actual goal blockage for women with children (range 0-44, $\mathrm{SD}=7.88$ ) than women without children (range $0-16, \mathrm{SD}=4.74$ ). Longitudinal analyses were conducted to assess whether baseline goal adjustment measures predicted a change in psychological adjustment measures for both groups. A significant interaction between group and general goal engagement emerged as a predictor of change in depressive symptoms. In subgroup analyses, general goal engagement was negatively associated with depressive symptoms for women without children and positively associated with depressive symptoms for women with children, although neither relationship was significant $(P<.10)$.

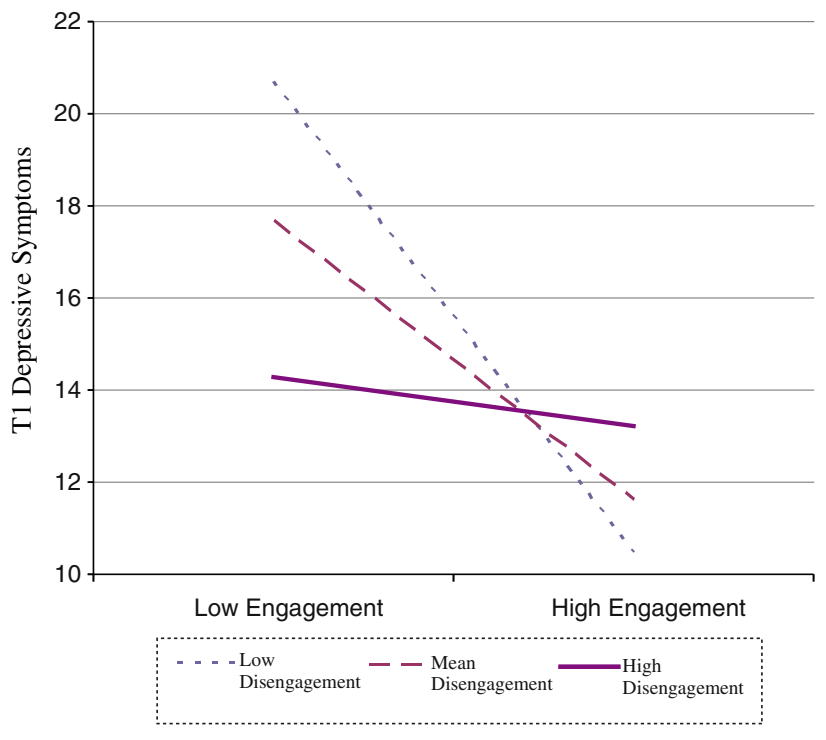

Fig. 1 T1 depressive symptoms regressed on goal disengagement at two levels of general goal engagement 


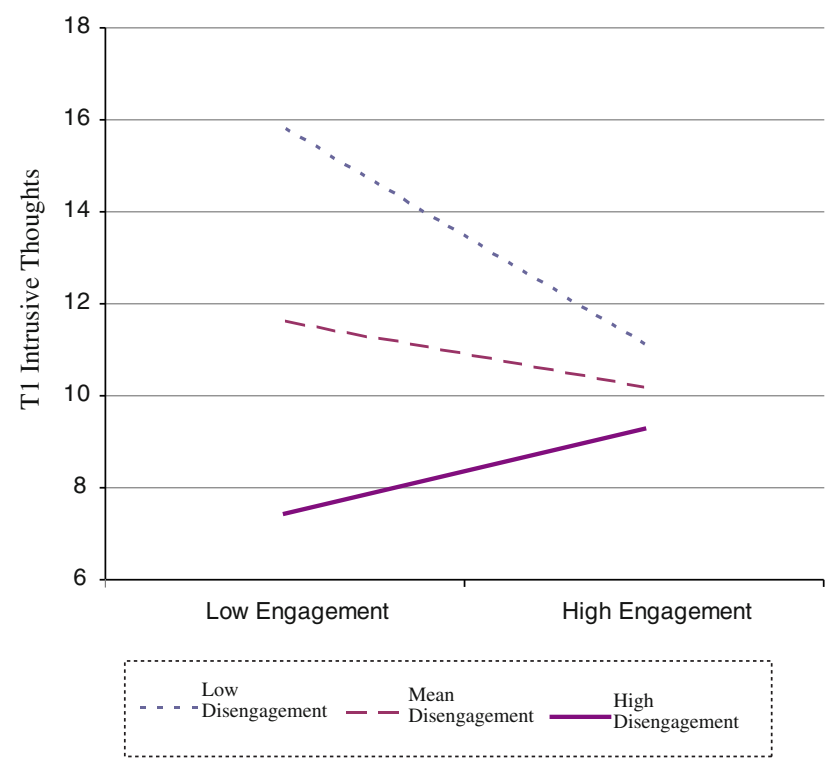

Fig. 2 T1 intrusive thoughts regressed on goal disengagement at two levels of general goal engagement

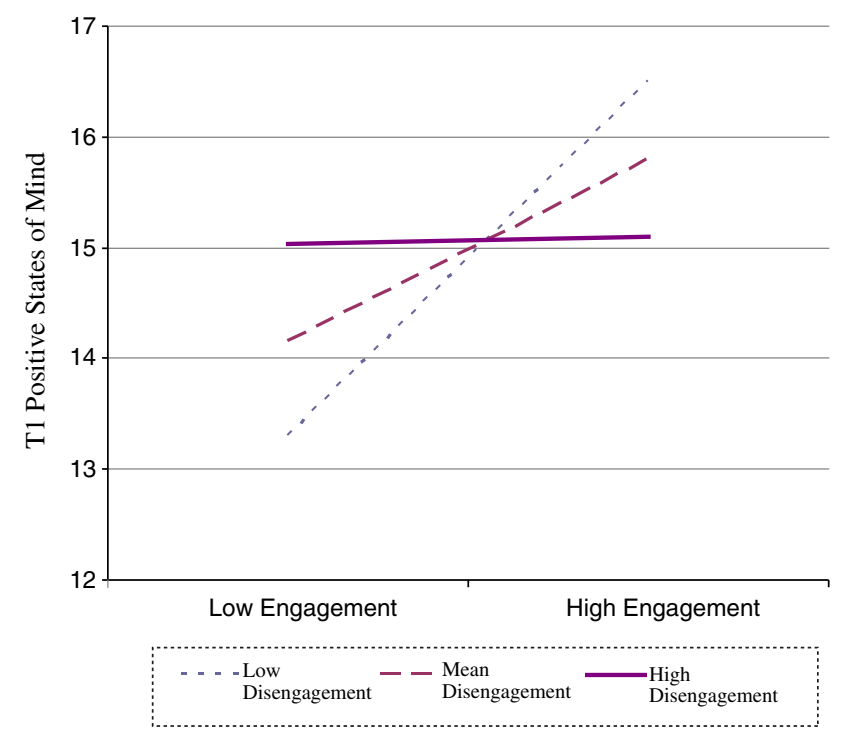

Fig. 3 T1 positive states of mind regressed on goal disengagement at two levels of general goal engagement

Psychological variables as predictors of change in goal adjustment abilities

$T$-tests, $\chi^{2}$ (for categorical variables) and correlations (for continuous variables) with the goal adjustment variables were used to select demographic and infertility-related variables for use in regression analyses. Experiencing a miscarriage $(t[37]=-2.45, P<.05)$ was associated with greater goal engagement in direct response to infertility at $\mathrm{T} 2$ and was included as a covariate in analyses. Regression analyses were conducted to examine CES-D, infertility-specific intrusive thoughts and PSOM scores as predictors of change in goal adjustment abilities. Significant covariates and T1 goal adjustment measures were entered in Step 1, and T1 psychological adjustment measures (CES-D, intrusive thoughts, PSOM) were entered in Step 2 in three separate regression models as displayed in Table 5. Baseline goal disengagement ability accounted for $47 \%$ of the variation in $\mathrm{T} 2$ goal disengagement ability, and psychological adjustment variables accounted for an additional $7-12 \%$ of the variance in $\mathrm{T} 2$ goal disengagement ability $(P<.01$ to $P<.001)$. Greater depressive symptoms at $\mathrm{T} 1$ were significantly associated with a decrease in goal disengagement ability $F(2,43)=31.10, P<.001$, as was T1 intrusive thoughts $F(2,43)=26.89, P<.001$. Greater positive states of mind at $\mathrm{T} 1$ were significantly associated with an increase in goal disengagement $F(2,43)=$ $24.41, P<.001$.

Similar regression analyses were conducted to examine the relationships between T1 perceived and actual goal blockage as predictors of change in goal adjustment abilities (Table 5). T1 goal adjustment measures were entered in Step 1 and Perceived and Actual Goal Blockage were entered together in Step 2. Greater Actual Goal Blockage at $\mathrm{T} 1$ was related to a decrease in goal disengagement ability, while greater Perceived Goal Blockage was associated with an increase in goal disengagement. Perceived and Actual Goal Blockage together accounted for $13 \%$ of the variance in T2 goal disengagement $F(3,40)=19.55, P<.001$, over and above the contribution of $\mathrm{T} 1$ goal disengagement.

None of the psychological adjustment variables predicted significant change in goal engagement in direct response to infertility over and above covariates and baseline levels of ability. However, higher T1 depressive symptoms and intrusive thoughts scores were significantly associated with a decrease in general goal engagement (Table 6). Baseline depressive symptoms explained 5\% of the variation of change in general goal engagement $F(2,43)=24.41, P<.001$, and intrusive thoughts scores explained $10 \%$ variation of change in general goal engagement $F(2,43)=29.78, P<.001$. Baseline positive states of mind, actual goal blockage, and perceived goal blockage were not significant predictors of change in general goal engagement scores when controlling for study entry ability and covariates. ${ }^{2}$

\footnotetext{
${ }^{2}$ Longitudinal analyses were conducted to assess whether baseline psychological measures predicted a change in goal adjustment measures for both women with children and women without children. T1 psychological functioning significantly predicted change in goal disengagement for women with children but did not predict significant change in goal disengagement for women without children. These relationships may be due to differences in variance in T2 goal disengagement for women with children (range 9-53, SD = 12.32) and women without children (range $9-44, \mathrm{SD}=8.69$ ). Interactions were
} 
Table 5 Predicting change in goal disengagement from psychological adjustment and goal blockage at study entry

\begin{tabular}{|c|c|c|c|c|c|}
\hline Predictor & Step & $R^{2} \Delta$ & $B$ & $\mathrm{SE}(B)$ & $\beta$ (final) \\
\hline T1 GD & 1 & $.47 * * *$ & 0.54 & 0.09 & $.69 * *$ \\
\hline CESD & 2 & $.11 * *$ & -0.25 & 0.08 & $-.33 * *$ \\
\hline T1 GD & 1 & $.47 * * *$ & 0.54 & 0.09 & $.69 * * *$ \\
\hline INT & 2 & $.08 * * *$ & -0.31 & 0.11 & $-.29 * * *$ \\
\hline T1 GD & 1 & $.47 * * *$ & 0.54 & 0.09 & $.69 * * *$ \\
\hline PSOM & 2 & $.12 * *$ & 0.99 & 0.28 & $.35 * *$ \\
\hline T1 GD & 1 & $.45 * * *$ & 0.52 & 0.09 & $.68 * * *$ \\
\hline Actual goal blockage & 2 & $.13 * *$ & -0.29 & 0.13 & $-.22 *$ \\
\hline Perceived goal blockage & & & 1.48 & 0.60 & $.26^{*}$ \\
\hline
\end{tabular}

$n=46$

CESD Center for Epidemiologic Studies-Depression Scale, INT Infertility-specific thought intrusion as measured by IES (Intrusion subscale), PSOM Positive States of Mind scale. $R$ reported is adjusted $R^{2}$

$* P<.05 ; * * P<.01 ; * * * P<.001$

Table 6 Predicting change in general goal engagement from psychological adjustment and goal blockage at study entry

\begin{tabular}{lllrrr}
\hline Predictor & Step & $R^{2} \Delta$ & $B$ & SE $(B)$ & $\beta$ (final) \\
\hline T1 GRGEN & 1 & $.47^{* * *}$ & 0.63 & 0.10 & $.70^{* * *}$ \\
CESD & 2 & $.05^{*}$ & -0.08 & 0.04 & $.23^{*}$ \\
T1 GRGEN & 1 & $.47^{* * *}$ & 0.63 & 0.10 & $-70^{* * *}$ \\
INT & 2 & $.10^{* * *}$ & -0.16 & 0.05 & $.32^{* * *}$ \\
T1 GRGEN & 1 & $.47^{* * *}$ & 0.63 & 0.10 & $.70^{* *}$ \\
PSOM & 2 & .02 & 0.19 & 0.16 & .14 \\
T1 GRGEN & 1 & $.51^{* * *}$ & -0.08 & 0.07 & -.10 \\
Actual goal blockage & 2 & .03 & -0.43 & & 0.31 \\
Perceived goal blockage & & & & -.15 \\
\hline
\end{tabular}

$n=46$

CESD Center for Epidemiologic Studies-Depression Scale; INT Infertility-specific thought intrusion as measured by IES (Intrusion subscale), $P S O M$ Positive States of Mind scale. $R$ reported is adjusted $R^{2}$

$* P<.05 ; * * P<.01 ; * * * P<.001$

\section{Discussion}

One key finding of this study is that in the context of infertility, strong engagement in pre-existing goals can buffer the relations of the inability to disengage from the goal of biological parenthood with poorer adjustment, as indicated by both negative psychological indicators (i.e., depressive symptoms, infertility-related intrusive thoughts) and a positive psychological indicator (i.e., positive states of mind). Regression models with goal-related variables accounted for $18-22 \%$ of the variance in $\mathrm{T} 1$ depressive symptoms, intrusive thoughts, and positive states of mind.

Previous studies have shown that high dispositional goal reengagement buffers against the negative effects of low

Footnote 2 continued not significant in predicting changes in general goal engagement or goal engagement in direct response to infertility. dispositional goal disengagement ability on a variety of outcomes including self-mastery, perceived stress, life satisfaction, self-reported cold symptoms (Wrosch et al. 2003b, 2007), and the current study provides support for this interaction in a specific situational context. Findings suggest that, for those individuals who have the most difficult time letting go of a cherished goal, promoting engagement with other valued goals may be a more potent intervention for bolstering mood than attempting to foster goal disengagement.

In light of emerging evidence that goal disengagement and reengagement have distinct relationships with negative versus positive indicators of adjustment (Thompson and Stanton 2009; Wrosch 2011; Wrosch et al. in press), we conducted analyses with separate dependent variables. However, the dependent variables were highly correlated in this sample, perhaps reflecting the tendency of negative and positive affect to become increasingly inversely related 
during stressful experiences (Zautra 2003; Zautra et al. 2002). Moreover, general goal engagement was protective in the context of low disengagement ability for each psychological adjustment variable. Future research should employ measures of both positive and negative psychological adjustment to assess whether goal disengagement is uniquely associated with fewer negative states and goal engagement is uniquely associated with greater positive states.

Although studies in which goal constructs are conceptualized as dispositional tendencies have found that goal engagement in direct response to goal blockage is adaptive (Wrosch et al. 2003b, 2008), a second key finding of the present study is that, in a specific situational context, general engagement with other goals is more strongly associated with psychological adjustment than is goal engagement in direct response to the blocked goal. Devoting attention to valued, pre-existing pursuits is likely to require less effort and to be more immediately satisfying than generating new goals or deliberately resurrecting former goals because the primary goal is blocked. The two processes also may be distinguished by the motivations behind pursuing alternative goals in parenthood. In the case of general goal engagement, the motivation for pursuing goals may be spontaneous or natural, in contrast to deliberate and effortful goal pursuit in response to the loss and trauma of infertility. Calculated goal pursuit in response to infertility may be more cognitively and emotionally taxing than continued goal engagement, as it may require identification and initiation of new goal pursuit rather than maintenance of a familiar goal. Another possibility is that the data fail to capture long-term benefits of adopting new goals in direct response to infertility, as the current study covered only a six-month time period. Perhaps pursuing a goal in response to a significant stressor may foster a longterm sense of purpose and satisfaction as progress is made towards an intentionally adopted goal. The current findings suggest the distinction between general goal engagement and goal engagement in direct response to goal blockage is an important one that requires longitudinal study.

A third key finding of this study is that high perceived and actual goal blockage were related to poorer adjustment across the three adjustment indicators, together explaining $7-11 \%$ of variance in psychological adjustment (with the exception of a nonsignificant relation between actual goal blockage and depressive symptoms). Interestingly, perceived goal blockage and actual goal blockage were uncorrelated and were both unique predictors of psychological adjustment, underlining the importance of assessing both objective and subjective indicators of goal blockage. Verhaak et al. (2006) posit that the strain of undergoing medical treatments (i.e. actual goal blockage) is distinct from the strain of risk of failure (i.e. perceived goal blockage), and that these two aspects of infertility have unique consequences for adjustment.

A fourth key finding involves the important role of psychological variables as a contributor to changes in goal adjustment over time. Poorer psychological adjustment as indicated by all three measures predicted a decrease in goal disengagement ability, and more depressive symptoms and intrusive thoughts predicted a decrease in general engagement with life goals. Wrosch and Miller (2009) found in a sample of adolescent females that higher depressive symptoms predicted an increase in goal disengagement ability. They surmised, however, that once goal adjustment abilities have reached relative stability in adulthood, depressive symptoms may actually hinder individuals' efforts at goal disengagement. Our data support this developmental perspective. Post hoc analyses revealed that the effect of baseline psychological adjustment on goal adjustment was primarily driven by women with children, such that poor baseline psychological adjustment predicted a decrease in goal adjustment ability only for women with children. While this finding could be explained statistically (due to the greater variance in $\mathrm{T} 2$ goal disengagement for women with children), future research should consider potential differences between women experiencing infertility before or after having a child or children.

As hypothesized, greater perceived goal blockage predicted an increase in goal disengagement ability. Contrary to hypothesis, actual goal blockage predicted a decrease in goal disengagement ability. This may represent a "digging in of one's heels," or a sense of entrapment based upon an assessment of sunk cost, as individuals invest more emotionally, physically, and financially with each failed treatment attempt. Overall, these intriguing findings underscore the importance of examining the severity of goal blockage in situational contexts.

Clinical implications of the findings include the value of assessing general goal engagement in addition to goal disengagement and goal engagement in response to goal blockage. Although clinicians may be inclined to focus on an identified stressor, like infertility, and the individual's response to that particular stressor, assessing and promoting life goals in unrelated domains may buffer against the negative psychological effects associated with goal blockage. Goal disengagement in conjunction with general goal engagement appears adaptive in the context of a chronic health stressor such as infertility; specific interventions designed to facilitate goal adjustment and promote general goal engagement should be developed and assessed. In that depressive symptoms and intrusive thoughts may constitute psychological risk factors that predict difficulty adjusting to goal blockage, targeting individuals experiencing high depressive symptoms and stressor-specific thought intrusion for intervention may prove particularly important. 
These data also underscore the importance of assessing perceived goal blockage, instead of drawing conclusions about goal blockage solely from treatment history or other observable markers of goal blockage. Assessing how an individual has arrived at his or her perception of goal attainability may aid clinicians in addressing cognitive distortions and promoting realistic appraisals of goal attainability.

This study's findings require consideration in light of its limitations. First, only heterosexual, partnered, and treatment-seeking women were included. Future research on response to infertility should incorporate diverse groups to expand generalizability and to examine potential differences across subsamples. As post hoc analyses revealed some significant differences between women with children and women without children, future research should also consider potential differences between these two subsamples of women. Second, the psychological adjustment variables examined demonstrated minimal change over a 6 month period, perhaps due to the chronic nature of the stressor. T1 goal adjustment variables were significantly correlated with T2 psychological adjustment variables, but were not sufficiently potent relative to the stability of the dependent variables to predict change. Future work should examine goal adjustment in response to a discrete stressor where greater change in psychological adjustment variables may be observed (e.g., see Berghuis and Stanton 2002, for a longitudinal examination of adjustment to a discrete, infertilityrelated, dyadic stressor). Third, as a consequence of attrition and achieved pregnancies, the small sample limited the power to detect significant effects longitudinally. Although completers and non-completers did not differ significantly on demographic or fertility-related variables, we suspect that non-completers might be more likely to have gotten pregnant than completers, owing to the low pregnancy rate in completers (11\%). Fourth, using a single item to assess perceived goal blockage reduced participant burden, but it might benefit from measurement through additional items. Finally, participants may have underreported goal disengagement ability due to unrealistic fear of decreasing their chances of becoming pregnant if they admitted to giving up while still pursuing treatment (K. J. Petrie, personal communication, October 7, 2009). Complementing self-report measures of goal adjustment and psychological adjustment with behavioral data and observational data from different sources (e.g., partner or doctor) would strengthen the methodology of future studies and enable assessment of biases in reporting goal adjustment abilities.

Further research should assess factors that may promote or impede goal adjustment, such as cognitive processes underlying goal adjustment, as well as the impact of situational factors such as perceived investment in the goal, attributions about causes of goal blockage, perceived conflict with other goals, and perceived consequences of goal failure. Important social factors influencing goal adjustment could include perceptions of goal-specific support from others, perceptions of normative pressures, and perceptions of others' assessment of goal importance and attainability. Although a dispositional measure of goal adjustment (Wrosch et al. 2003b) was not available when these data were collected, the influence of dispositional factors on situational goal blockage is a promising future research focus.

Future research should explore which cognitive and emotional processes facilitate or impede adaptive goal adjustment, and how these cognitive processes can be altered through intervention. For example, cognitive disengagement from goals may occur through mechanisms such as emotional acceptance and adequate explanation of the goal blockage. Data demonstrate that how individuals evaluate their response to stressful life experiences and how they explain such events are significant predictors of adjustment (Low et al. 2008; Wilson and Gilbert 2008). Previous research has identified specific cognitive styles, such as intellectual reflection and angry rumination, which are associated with attentional inflexibility and may characterize individuals with low goal disengagement ability (Pyszczynski and Greeenberg 1987; Whitmer and Banich 2007). Examining the roles of cognitive styles, emotional processing, and other mechanisms in goal adjustment may advance theories on adaptive goal adjustment and provide grounding for effective interventions.

In summary, these findings support the theory that goal disengagement is adaptive in the context of profound goal blockage and make an important contribution by suggesting that engagement with pre-existing goals may be more protective than deliberate engagement with new or former goals motivated by or in direct response to goal blockage. Depressive symptoms and intrusive thoughts may be notable risk factors that predict inflexible goal pursuit in the context of infertility and other medical stressors. Understanding the relationships between goal adjustment and psychological adjustment has the potential to inform interventions that may reduce distress and improve psychological well-being for individuals confronting chronic medical conditions.

Open Access This article is distributed under the terms of the Creative Commons Attribution Noncommercial License which permits any noncommercial use, distribution, and reproduction in any medium, provided the original author(s) and source are credited.

\section{References}

Abma, J. C., Chandra, A., Mosher, W. D., Peterson, L. S., \& Piccinino, L. J. (1997). Fertility, family planning, and women's 
health: New data from the 1995 national survey of family growth. National Center for Health Statistics. Vital and Health Statistics, 23, 1-114.

Barnett, R. C. (2004). Women and multiple roles: Myths and realities. Harvard Review of Psychiatry, 12, 158-163.

Berghuis, J. P., \& Stanton, A. L. (2002). Adjustment to a dyadic stressor: A longitudinal study of coping and depressive symptoms in infertility couples over an insemination attempt. Journal of Consulting and Clinical Psychology, 70, 433-438.

Carver, S. C., \& Scheier, M. F. (2000). Scaling back goals and recalibration of the affect system are processes normal in adaptive self-regulation: Understanding the 'response shift' phenomena. Social Science and Medicine, 50, 1715-1722.

Clark, L. F., Henry, S. M., \& Taylor, D. M. (1991). Cognitive examination of motivation for childbearing as a factor in adjustment to infertility. In A. L. Stanton \& C. Dunkel-Schetter (Eds.), Infertility: Perspectives from stress and coping research (pp. 157-180). New York: Plenum Press.

Cordova, M. J., Cunningham, L. L., Carlson, C. R., \& Andrykowski, M. A. (2001). Posttraumatic growth following breast cancer: A controlled comparison study. Health Psychology, 20, 176-185.

Dougher, M. J. (1994). The act of acceptance. In S. C. Hayes, N. S. Jacobson, V. M. Follette, \& M. J. Dougher (Eds.), Acceptance and change: Content and context in psychotherapy (pp. 37-45). Reno: Context Press.

Frederickson, B. L. (2001). The role of positive emotions in positive psychology. The broaden-and-build theory of positive emotions. American Psychologist, 56, 218-226.

Greil, A. L. (1997). Infertility and psychological distress: A critical review of the literature. Social Science and Medicine, 45, $1679-1700$.

Heckhausen, J., Wrosch, C., \& Schulz, R. (2010). A motivational theory of life-span development. Psychological Review, 117, $32-60$.

Hill, J. A. (1995). T-helper 1-type immunity to trophoblast: Evidence for a new immunological mechanism for recurrent abortion in women. Human Reproduction, 10, 114-120.

Horowitz, M., Adler, N., \& Kegeles, S. (1988). A scale for measuring the occurrence of positive states of mind: A preliminary report. Psychosomatic Medicine, 50, 477-483.

Horowitz, M. J., Wilner, N., \& Alvarez, W. (1979). Impact of event scale: A measure of subjective distress. Psychosomatic Medicine, 41, 209-218.

Kraaij, V., Garnefski, N., \& Schroevers, M. J. (2009). Coping, goal adjustment, and positive and negative affect in definitive infertility. Journal of Health Psychology, 14, 18-26.

Linville, P. W. (1987). Self-complexity as a cognitive buffer against stress-related illness and depression. Journal of Personality and Social Psychology, 52, 663-676.

Low, C. A., Stanton, A. L., \& Bower, J. E. (2008). Effects of acceptance-oriented versus evaluative emotional processing on heart rate recovery and habituation. Emotion, 8, 419-924.

Miller, G. E., \& Wrosch, C. (2007). You've gotta know when to fold 'em: Goal disengagement and systemic inflammation in adolescence. Psychological Science, 18, 773-777.

Mosher, W. D., \& Pratt, W. F. (1982). Reproductive impairments among married couples: United States. Maryland: U.S. Department of Health and Human Services, Office of Health Research, Statistics, and Technology, National Center for Health Statistics.

Niedenthal, P. M., Setterlund, M. B., \& Wherry, M. B. (1992). Possible self-complexity and affective reactions to goal relevant evaluation. Journal of Personality and Social Psychology, 63, 5-16.

Pyszczynski, T., \& Greeenberg, J. (1987). Self-regulatory preservation and the depressive self-focusing style: A self-awareness theory of reactive depression. Psychological Bulletin, 102, $122-138$.

Radloff, L. S. (1977). The CES-D scale: A self-report depression scale for research in the general population. Applied Psychological Measurement, 1, 385-401.

Radloff, L. S. (1991). The use of the center for epidemiologic studies depression scale in adolescents and young adults. Journal of Youth and Adolescence, 20, 149-166.

Ruderman, M. N., Ohlott, P. J., Panzer, K., \& King, S. (2002). Benefits of multiple roles for managerial women. Academy of Management Journal, 45, 369-386.

Salmela-Aro, K., \& Suikkari, A.-M. (2008). Letting go of your dreams-adjustment of child-related goal appraisals and depressive symptoms during infertility treatment. Journal of Research in Personality, 42, 988-1003.

Sieber, S. D. (1974). Toward a theory of role accumulation. American Sociological Review, 39, 567-578.

Thompson, E. H., \& Stanton, A. L. (2009, April). Giving up life goals in the context of metastatic breast cancer. Poster presented at the annual meeting of the Society of Behavioral Medicine, Montreal, ON

Verhaak, C. M., Smeenk, J. M. J., Evers, A. W. M., Kremer, J. A. M., \& Kraaimaat, F. W. (2006). Women's emotional adjustment to IVF: A systematic review of 25 years of research. Human Reproduction Update, 13, 27-36.

Whitmer, A. J., \& Banich, M. T. (2007). Inhibition versus switching deficits in different forms of rumination. Psychological Science, $18,546-553$.

Wilson, T. D., \& Gilbert, D. T. (2008). Explaining away: A model of affective adaptation. Perspectives on Psychological Science, 3, 370-381.

Woodward, J. T. (2004). Acceptance and regoaling in the context of infertility: How do we move forward during blockage of a major life goal? (Doctoral dissertation, U of Kansas, August 2004). Dissertation Abstracts International, 65, 3153193.

Wrosch, C. (2011). Self-regulation of unattainable goals and pathways to quality of life. In S. Folkman (Ed.), The Oxford handbook of stress, health, and coping (pp. 319-333). New York: Oxford University Press.

Wrosch, C., \& Miller, G. F. (2009). Depressive symptoms can be useful: Self-regulatory and emotional benefits of dysphoric mood in adolescence. Journal of Personality and Social Psychology, 96, 1181-1190.

Wrosch, C., Miller, G. F., Scheier, M. F., \& de Pontet, S. B. (2007). Giving up on unattainable goals: Benefits for health? Personality and Social Psychology Bulletin, 33, 251-263.

Wrosch, C., Scheier, M. F., Carver, C. S., \& Schulz, R. (2003a). The importance of goal disengagement in adaptive self-regulation: When giving up is beneficial. Self and Identity, 2, 1-20.

Wrosch, C., Scheier, M. F., Miller, G. E., \& Carver, C. S. (in press). When meaning is threatened: The importance of goal adjustment for psychological and physical health. In P. T. Wong (Ed.), The human quest for meaning. Routledge.

Wrosch, C., Scheier, M. F., Miller, G. E., Schulz, R., \& Carver, C. S. (2003b). Adaptive self-regulation of unattainable goals: Goal disengagement, goal reengagement, and subjective well-being. Personality and Social Psychology Bulletin, 29, 1494-1508.

Zautra, A. J. (2003). Emotions, stress, and health. New York: Oxford University Press.

Zautra, A. J., Berkhof, J., \& Nicolson, N. A. (2002). Changes in affect interrelations as a function of stressful events. Cognition and Emotion, 16, 309-318. 